\title{
Enthusiasm cools in Q2
}

Stacy Lawrence

Although private financing and overall funding remain relatively stable, investor interest in biotech is a shadow of a year ago, when the initial public offering (IPO) market had just come off the best

\section{Biotech stock market performance}

Biotech indices from BioCentury and Nasdaq have declined by $>10 \%$ since Q2 2004.

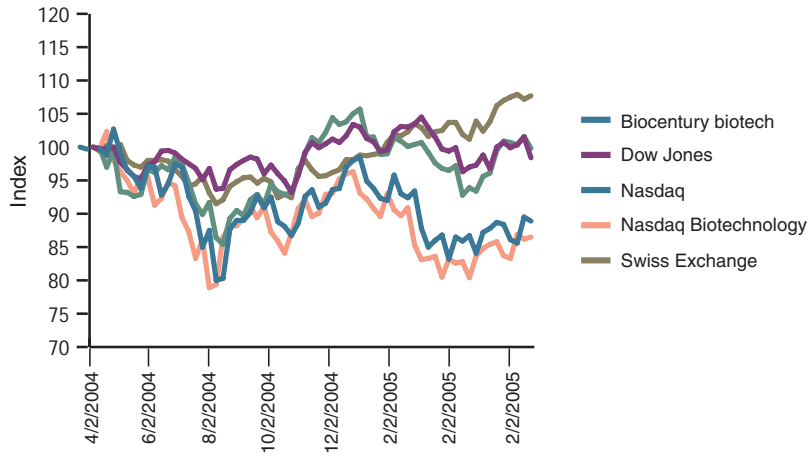

Source: Multex, BioCentury

\section{Global biotech venture capital investment}

As they have for the past five quarters, venture capital totals held relatively steady in the second quarter.

$\square$ North America

$\square$ Europe

$\square$ Asia-Pacific

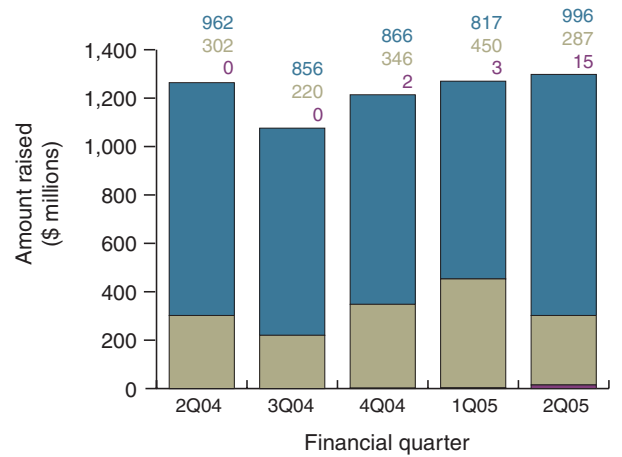

Source: BioCentury

\section{Global biotech industry financing}

With partnering revenue up, overall funding during Q2 exceeded the total amount in the same quarter last year.

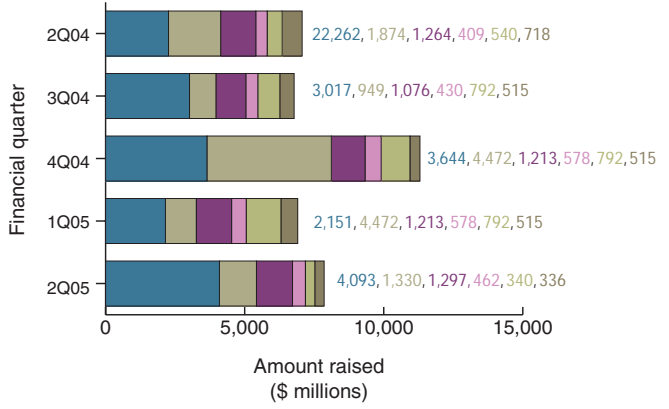

Source: BioCentury, Burrill \& Company by a little more than $10 \%$, and the NASDAQ Biotechnology Index has receded by almost $15 \%$. Public offerings and follow-on financing have dwindled from the high early last year, even with a robust IPO market in Europe.

\section{Global biotech initial public offerings}

With six public offerings, European IPO fundraising totals outstripped those in the US for the first time during this IPO window.

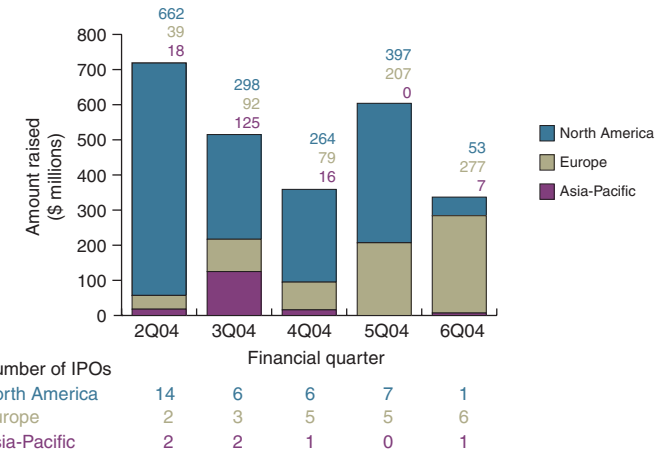

Source: BioCentury

\begin{tabular}{|c|c|c|c|c|}
\hline & Company (lead underwriter) & $\begin{array}{l}\text { Amount raised } \\
\text { ( } \$ \text { millions })\end{array}$ & $\begin{array}{l}\text { Percent } \\
\text { change in } \\
\text { stock price } \\
\text { since offer }{ }^{1}\end{array}$ & $\begin{array}{l}\text { Date } \\
\text { launched }\end{array}$ \\
\hline \multirow[t]{6}{*}{ IPOs } & Arpida (Deutsche Bank) & $\$ 81$ & $-8 \%$ & 4-May \\
\hline & $\begin{array}{l}\text { ProStrakan Group (Morgan } \\
\text { Stanley) }\end{array}$ & $\$ 73$ & $3 \%$ & 10-Jun \\
\hline & XenoPort (Morgan Stanley) & $\$ 53$ & $2 \%$ & 2-Jun \\
\hline & TopoTarget (N/A) & $\$ 37$ & $3 \%$ & 9-Jun \\
\hline & Devgen (KBC Securities) & $\$ 37$ & $5 \%$ & 7-Jun \\
\hline & Company (lead investor) & $\begin{array}{l}\text { Amount invested } \\
\text { (\$ millions) }\end{array}$ & $\begin{array}{l}\text { Round } \\
\text { number }\end{array}$ & Date closed \\
\hline \multirow[t]{9}{*}{$\begin{array}{l}\text { Venture } \\
\text { capital }\end{array}$} & $\begin{array}{l}\text { Verus Pharmaceuticals (Domain } \\
\text { Associates, Prospect Venture } \\
\text { Partners) }\end{array}$ & $\$ 78$ & 1 & 9-Jun \\
\hline & $\begin{array}{l}\text { Somaxon Pharmaceuticals } \\
\text { (MPM Capital) }\end{array}$ & $\$ 65$ & 3 & 6-Jun \\
\hline & Oxagen (MPM Capital) & $\$ 60$ & 2 & 23-May \\
\hline & $\begin{array}{l}\text { Gemin X Biotechnologies } \\
\text { (H.I.G. Ventures) }\end{array}$ & $\$ 50$ & 4 & 19-May \\
\hline & $\begin{array}{l}\text { Osiris Therapeutics (Friedli } \\
\text { Corporate Finance) }\end{array}$ & $\$ 50$ & N/A & 8-Jun \\
\hline & Azur Pharma (N/A) & $\$ 49$ & $N / A$ & 8-Jun \\
\hline & $\begin{array}{l}\text { Elbion (3i, Burrill \& Company, } \\
\text { Deutsche Venture Capital) }\end{array}$ & $\$ 45$ & 1 & 24-May \\
\hline & Target & Acquirer & $\begin{array}{l}\text { Value } \\
\text { (\$ millions) }\end{array}$ & $\begin{array}{l}\text { Date } \\
\text { announced }\end{array}$ \\
\hline & Vicuron & Pfizer & $\$ 1,900$ & 15-Jun \\
\hline \multirow[t]{5}{*}{$\begin{array}{l}\text { Mergers and } \\
\text { acquisitions }\end{array}$} & Transkaryotic Therapies & $\begin{array}{l}\text { Shire } \\
\text { Pharmaceuticals }\end{array}$ & $\$ 1,600$ & 21-Apr \\
\hline & Bone Care & Genzyme & $\$ 719$ & 4-May \\
\hline & Corixa & GlaxoSmithKline & $\$ 300$ & 2-May \\
\hline & Peninsula Pharmaceuticals & Johnson \& Johnson & $\$ 245$ & 19-Apr \\
\hline & Researcher & Investor & $\begin{array}{l}\text { Value } \\
\text { (\$ millions) }\end{array}$ & Deal type \\
\hline \multirow[t]{6}{*}{$\begin{array}{l}\text { Licensing / } \\
\text { collaboration }\end{array}$} & Karlin Technology & Medtronic & $\$ 800$ & $\begin{array}{l}\text { Asset pur- } \\
\text { chase }\end{array}$ \\
\hline & Anadys Pharmaceuticals & Novartis & $\$ 570$ & $\begin{array}{l}\text { Co-develop- } \\
\text { ment, license }\end{array}$ \\
\hline & Alkermes & Cephalon & $\$ 490$ & License \\
\hline & Biogen IDEC & Genentech & $\$ 408$ & $\begin{array}{l}\text { Asset pur- } \\
\text { chase }\end{array}$ \\
\hline & Arakis, Vectura & Novartis & $\$ 375$ & Collaboration \\
\hline & BioMarin Pharmaceuticals & Serono & $\$ 257$ & Collaboration \\
\hline
\end{tabular}

